\title{
Using Bootstrap Method to Evaluate the Power of Tests for Non-Linearity in Asymmetric Price Relationship
}

\author{
Henry De-Graft Acquah \\ University of Cape Coast, Cape Coast, Ghana \\ henrydegraftacquah@yahoo.com
}

\begin{abstract}
This paper introduces and applies the bootstrap method to compare the power of the test for asymmetry in the Granger and Lee (1989) and Von Cramon-Taubadel and Loy (1996) models. The results of the bootstrap simulations indicate that the power of the test for asymmetry depends on various conditions such as the bootstrap sample size, model complexity, difference in adjustment speeds and the amount of noise in the data generating process used in the application. The true model achieves greater power when compared with the complex model. With small bootstrap sample size or large noise, both models display low power in rejecting the (false) null hypothesis of symmetry.
\end{abstract}

Keywords: Bootstrap Method; Asymmetry; Model Complexity; Power Test

\section{Introduction}

Previous studies investigating asymmetric price transmission have demonstrated that the Granger and Lee, and Von Cramon-Taubadel and Loy models have low power in rejecting the incorrect null hypothesis of symmetry. For example, Acquah and Von Cramon -Taubadel (2009) examined the power of the Granger and Lee (1989), and Von Cramon-Taubadel and Loy (1996) models of asymmetry via Monte Carlo experimentation and found them to have low power. They also noted that the power of the test for asymmetry depends on various conditions such as sample size, model complexity, difference in adjustment speeds and the amount of noise in the data generating process used in the application. However, Acquah and Von Cramon -Taubadel (2009) did not consider the use of an alternative simulation technique such as bootstrap method to evaluate the power of the test for non-linearity in the Granger and Lee, and Von Cramon-Taubadel and Loy models. Though the bootstrap method offers an advantage over the previous Monte Carlo technique which makes implicit assumption about the distribution and the true values of the parameters. Additionally, very little is known about the usefulness of the bootstrap method as a tool for evaluating the power of Granger and Lee, and Von Cramon-Taubadel and Loy models. However, the bootstrap technique could be considered as alternative approach to evaluating the power of the test for asymmetry. Fundamentally, this study fills the gap in the literature by introducing the bootstrap approach and employing it to evaluate the power of the Granger and Lee, and Von Cramon-Taubadel and Loy models. The purpose of this paper is therefore to support the claim that the Granger and Lee, and Von Cramon-Taubadel and Loy models have low power and in so doing demonstrate the use of bootstrap method to evaluate the power of the test for asymmetry in these models. Unlike previous studies, I depart from Monte Carlo experimentation and implement bootstrap algorithm to evaluate the power of the test for asymmetry.

\section{Literature Review}

Asymmetry may be defined as an unreciprocal relationship between rises and falls in prices. For example, farm and retail prices. Asymmetric adjustment in the transmission of prices at various levels of the agricultural marketing system has been of considerable empirical interest to agricultural economists. Subsequently, numerous methods have been developed to measure price asymmetry. Earlier studies investigating price asymmetry emphasized the widely used Houck's (1977) approach. Recent studies have demonstrated that this approach tends to have low power. For example, Acquah (2010) in a Monte Carlo experimentation demonstrated that the failure of the Houck's model to capture asymmetry in practice is due to low power. Alternatively, Acquah (2012) in a bootstrap simulation finds the Houck's model to have low power in bootstrap samples. Furthermore, other studies note that the specification of the Houck's model does not take into consideration the time series properties of the data. This assertion provides a basis for the error correction modelling. The asymmetric Error Correction Model is motivated 
by the fact that the variants of the Houck's model are not consistent with cointegration between the price series.

Against this background, Engle and Granger (1987), notes that if two prices are cointegrated, then an error correction mechanism exist. Subsequently, Granger and Lee (1989) proposed a modification to error correction term that makes it possible to test for asymmetric price transmission. Numerous studies have applied variants of the Granger and Lee model. For instance, Von Cramon-Taubadel and Loy (1996) extended the Granger and Lee model to a complex asymmetric Error Correction model. This complex model differs from the Granger and Lee model with regards to the number of asymmetric adjustment parameters. Numerous studies have applied the Von Cramon-Taubadel and Loy model. However, some studies (Cook, Holly and Turner, 1999a and Acquah and Von Cramon-Taubadel, 2009) have found Granger and Lee, and Von Cramon-Taubadel and Loy model to have low power via Monte Carlo experimentation. The current study supports the claim that the Granger and Lee, and Von CramonTaubadel and Loy models have low power and demonstrates this using bootstrap method.

\section{Methodology}

The methodology describes the different methods of testing for asymmetry with emphasis on the Granger and Lee, and Von Cramon-Taubadel and Loy models. The bootstrap method is presented with emphasis on parametric bootstrap.

Different Methods of Testing for Asymmetry: The data generating process is derived from the Granger and Lee (1989) Error Correction Model and can be written as follows:

$$
\Delta y_{t}=\beta_{1} \Delta x_{t}+\beta_{2}(y-x)_{t-1}+\varepsilon_{2, t} \quad \varepsilon_{2, t} \sim N\left(0, \delta^{2}\right)
$$

Where $y$ and $x$ are generated as I (1) non stationary variables that are cointegrated and there exist an equilibrium relationship between $\mathrm{y}$ and $\mathrm{x}$ which is defined by an error correction term. The long run dynamics captured by the error correction term are implicitly symmetric. In order to incorporate asymmetric adjustments, the error correction term can be segmented into positive and negative components as follows:

$$
\begin{aligned}
& (y-x)_{t}^{+}=\left[\begin{array}{cc}
(y-x)_{t}, & \text { if } \\
\text { zero } & (y-x)_{t}>0 \\
\text { otherwise }
\end{array}\right. \\
& (y-x)_{t}^{-}=\left[\begin{array}{cc}
(y-x)_{t}, & \text { if }(y-x)_{t}<0 \\
\text { zero } & \text { otherwise }
\end{array}\right.
\end{aligned}
$$

The resulting asymmetric ECM is defined as

$$
\Delta y_{t}=\beta_{1} \Delta x_{t}+\beta_{2}^{+}(y-x)_{t-1}^{+}+\beta_{2}^{-}(y-x)_{t-1}^{-}+\varepsilon_{3, t} \quad \varepsilon_{3, t} \sim N\left(0, \delta^{2}\right)
$$

Asymmetry is incorporated by allowing the speed of adjustment to differ for the positive and negative components of the Error Correction Term since the long run relationship captured by the error correction term was symmetric. Symmetry in equation $(4)$ is tested by determining whether the coefficients $\left(\beta_{2}^{+}\right.$ and $\beta_{2}{ }^{-}$) are identical (that is $H_{0}: \beta_{2}^{+}=\beta_{2}^{-}$).

Von Cramon-Taubadel and Loy (1996) in a departure from Granger and Lee model proposes a complex approach to asymmetry in which asymmetries specified affects the direct impact of price increases and decreases as well as adjustments to the equilibrium level.

$$
\Delta y_{t}=\beta_{1}^{+} \Delta x_{t}+\beta_{1}^{-} \Delta x_{t}^{-}+\beta_{2}^{+}(y-x)_{t-1}^{+}+\beta_{2}^{-}(y-x)_{t-1}^{-}+\varepsilon_{4, t} \quad \varepsilon_{4, t} \sim N\left(0, \delta^{2}\right)
$$

Where $\Delta x_{t}^{+}$and $\Delta x_{t}^{-}$are the positive and negative changes in $x_{t}$ and the remaining variables are defined as in equation (4). 
A formal test of the asymmetry hypothesis using the above equation is: $\boldsymbol{H}_{\mathrm{O}}: \boldsymbol{\beta}_{1}{ }^{+}=\boldsymbol{\beta}_{1}{ }^{-}$and $\beta_{2}{ }^{+}=\beta_{2}{ }^{-}$. In this case, a joint F-test can be employed to determine symmetry or asymmetry of the price transmission process.

Bootstrap Method: Bootstrap is a computer intensive resembling technique for robust estimation of statistics of interest. The basic idea of the bootstrap involves repeated random sampling with replacement from the original data to produce random samples of the same size as the original sample. Each of the selected samples is referred to as a bootstrap sample and each provides an estimate of the parameter of interest. With replacement, it is possible that any observation can be sampled more than once in each bootstrap sample. With replacement suggest that although each resample will have the same number of elements as the original sample, it could include some of the original data points more than once and some not included. In effect, each of these resample's or bootstrap samples will randomly depart from the original sample. And since the elements in these resamples vary slightly, the statistic of interest calculated from these resamples take on slightly different values.

In summary, the steps of the bootstrap procedure can be stated as follows:

- Construct an empirical distribution from the sample.

- From the empirical distribution draw a random sample of size $n$ with replacement.

- Calculate the statistic of interest.

- Repeat steps 2 and 3 B times, where B is a large number in order to create the bootstrap resamples.

Bootstrapping Regression Models: The procedure of the previous section can be easily extended to regression models. Parametric bootstrapping in regression models can be outlined as follows:

1. Estimate the regression coefficients of the original sample and calculate the fitted value and the residual for each observation.

2. Select the bootstrap of the residuals by sampling with replacement from the residuals. Calculate the bootstrap outcome variable as follows

$y^{*}=x \hat{\beta}+\varepsilon^{*}$

Regress the bootstrap $y^{*}$ values on the fixed $\mathrm{x}$ values and compute the statistic of interest.

\section{Results and Discussion}

In order to investigate the power of the test for asymmetry under various conditions, a series of bootstrap comparison of the two competing models are carried out based on 10000 replications. Drawing upon the experimental design of Acquah and Von Cramon-Taubadel (2009), the data generation process is specified in equation (4) with $\beta_{1}$ set to 0.5 and $\left(\beta_{2}{ }^{+}, \beta_{2}{ }^{-}\right) \in(-0.25,-0.50) \operatorname{or}(-0.25,-0.75)$. However, we attempt to measure asymmetric price transmission using alternative approaches: the Von Cramon-Taubadel and Loy asymmetric ECM, and the Granger and Lee Model. The two methods are compared in terms of their ability to reject the (false) null of symmetric adjustment against the (true) alternative of asymmetric adjustment using an F-test of the restricted versus the unrestricted model. The results in Table 1 indicate the low power of the conventional F-test in rejecting the incorrect null hypothesis of symmetry. For the sake of brevity, we denote the Von Cramon-Taubadel and Loy ECM, and the Granger and Lee ECM by VCTL and GL respectively.

Table 1: Rejection frequencies based on 10000 Bootstrap Replications GL-ECM DGP Model Estimated GL-ECM VCTL-CECM

\begin{tabular}{lllllll}
$\begin{array}{l}\text { Sample } \\
\text { size }\end{array}$ & $\left(\beta_{2}^{+}, \beta_{2}^{-}\right)$ & $\begin{array}{l}\text { Error } \\
\text { Size }\end{array}$ & $\mathbf{5 \%}$ & $\begin{array}{c}\text { Rejection } \\
\mathbf{1 \%}\end{array}$ & $\begin{array}{c}\text { Frequencies } \\
\mathbf{5 \%}\end{array}$ & $\mathbf{1 \%}$ \\
\hline 50 & $(-0,25,-0.50)$ & 3 & 0.1177 & 0.0379 & 0.1066 & 0.0322 \\
50 & $(-0,25,-0.50)$ & 2 & 0.1288 & 0.0416 & 0.1160 & 0.0350 \\
50 & $(-0,25,-0.50)$ & 1 & 0.1750 & 0.0716 & 0.1486 & 0.0569
\end{tabular}




\begin{tabular}{lllllll}
150 & $(-0,25,-0.50)$ & 3 & 0.1431 & 0.0550 & 0.1304 & 0.0466 \\
150 & $(-0,25,-0.50)$ & 2 & 0.1837 & 0.0756 & 0.1584 & 0.0626 \\
150 & $(-0,25,-0.50)$ & 1 & 0.3488 & 0.1791 & 0.2938 & 0.1421 \\
& & & & & \\
500 & $(-0,25,-0.50)$ & 3 & 0.2158 & 0.1024 & 0.1879 & 0.0830 \\
500 & $(-0,25,-0.50)$ & 2 & 0.3295 & 0.1736 & 0.2857 & 0.1330 \\
500 & $(-0,25,-0.50)$ & 1 & 0.7279 & 0.5514 & 0.6562 & 0.4670 \\
50 & $(-0.25,-0.75)$ & 3 & 0.1423 & 0.0510 & 0.1235 & 0.0404 \\
50 & $(-0.25,-0.75)$ & 2 & 0.1751 & 0.0676 & 0.1513 & 0.0554 \\
50 & $(-0.25,-0.75)$ & 1 & 0.3658 & 0.1822 & 0.2955 & 0.1399 \\
150 & $(-0.25,-0.75)$ & 3 & & & & \\
150 & $(-0.25,-0.75)$ & 2 & 0.2264 & 0.1034 & 0.1955 & 0.0806 \\
150 & $(-0.25,-0.75)$ & 1 & 0.3489 & 0.1902 & 0.2963 & 0.1455 \\
500 & $(-0.25,-0.75)$ & 3 & 0.7709 & 0.6096 & 0.7058 & 0.5236 \\
500 & $(-0.25,-0.75)$ & 2 & & & & 0.435 \\
500 & $(-0.25,-0.75)$ & 1 & 0.7368 & 0.5503 & 0.6678 & 0.4685 \\
\hline
\end{tabular}

The Bootstrap simulation indicates the low power of the conventional F-test in rejecting the null of symmetric adjustment in small sample sizes. Noticeably, both the Granger and Lee, and the Von CramonTaubadel and Loy model display lower power in small bootstrap samples. Improvements in power are observed if we increase the difference in asymmetric adjustment parameters from 0.25 to 0.5 in the true model. Similarly, if we decrease the amount of noise in the data generating process (DGP) systematically, an increase in power is also observed in both the complex ECM and standard ECM approaches as illustrated in Table 1 . The true model displayed greater power when compared with an overparameterised or complex model. These results suggest that the over-parameterised model reduces the power of the test for asymmetry in bootstrap samples. These findings are consistent with Acquah and Von Cramon -Taubadel (2009) who examined the power of the Granger and Lee (1989), and the Von CramonTaubadel and Loy (1996) models of asymmetry via Monte Carlo experimentation and found them to have low power. The findings of this research indicates that bootstrap sample sizes, difference between the asymmetric adjustment parameters, model complexity (i.e. number of parameters) and the amount of noise in the data generating process are important in the power of the test for asymmetry. With small bootstrap sample size or large noise, both Granger and Lee (1989), and Von Cramon-Taubadel and Loy (1996) models display low power in rejecting the (false) null hypothesis of symmetry.

\section{Conclusion}

The power of alternative approaches of measuring asymmetry has been compared. Using bootstrap methods, it has been demonstrated that the alternative approaches detect asymmetry at different rates under various conditions given the same data generating process. The results of the bootstrap simulations indicate that rejection frequencies increase with increases in sample size, increases in the difference between the asymmetric adjustment speeds and decreases in the amount of noise in the true data generating process used in the application. The Granger and Lee asymmetric ECM specification has greater power when compared to the Von Cramon-Taubadel and Loy ECM, although the power test for both models tends to be low in small bootstrap samples. Notably, this research establishes that an overparameterized model decreases the power of the test for asymmetry in bootstrap samples. The result demonstrates the usefulness of the bootstrap methods as an alternative approach to investigating the power of the test for asymmetry. Future research will explore the use of non parametric bootstrap to evaluate the power of the test for asymmetry. 


\section{Reference}

Acquah, H. D. (2012). A Bootstrap Approach to Testing for Symmetry in the Granger and Lee Asymmetric Error Correction Model. Russian Journal of Agricultural and Socio-Economic Sciences, 11(11), 3336.

Acquah, H. D. (2010). Testing for Symmetry in the Houck's Model. Indian Development Review: An International Journal of Development Economics, 8(1), 105-107.

Acquah, H. D. \& Von Cramon-Taubadel, S. (2009). A Monte Carlo Comparison of the Power of Tests for Non-Linearity in Asymmetric Price Transmission Models. International Journal of Computational Intelligence Research and Application, 3(2), 281-283.

Cook, S., Holly, S. \& Turner, P. (1999a). The power of tests for non-linearity: the case of Granger-Lee asymmetry. Economics Letters, 62, 155-59.

Engle, R. F. \& Granger, C. W. J. (1987). Co-integration and error correction: Representation, estimation and testing. Econometrica, 55, 251-276.

Granger, C. W. J. \& Lee, T. H. (1989). Investigation of production, sales and inventory relationships using multicointegration and non-symmetric error correction models. Journal of Applied Econometrics, 4, 135-159.

Houck, J. P. (1977). An Approach to specifying and estimating nonreversible Functions. American Journal of Agricultural Economics, 59, 570-572.

Von Cramon-Taubadel, S. \& Loy, J. P. (1996). Price asymmetry in the international wheat market: Comment. Canadian Journal of Agricultural Economics, 44, 311-317. 